\title{
Homínidos, dudas y grandes titulares: La controversia del Hombre de Orce en la prensa española (1983-2007)
}

\section{Miquel Carandell Baruzzi (*)}

${ }^{*}$ Centre d'Història de la Ciència, CEHIC. Universitat Autònoma de Barcelona

Miquel.carandell@e-campus.uab.cat

Dynamis

[0211-9536] 2013; 33 (2): 365-387
Fecha de recepción: 7 de agosto de 2012

Fecha de aceptación: 28 de febrero de 2013

SUMARIO: 1.-Introducción. 2.-El Hombre de Orce: «El Hallazgo del Siglo» (o eso parece). 3.-Congreso Internacional de Paleontología Humana en Orce: «Josep Gibert tenía razón» (o eso parece). 4.-Ocho centímetros de polémica: características anatómicas de VM-0. 5.— «Una dimensión distinta»: los medios de comunicación y el Hombre de Orce. 5.1.-Divulgación y validación en la prensa. 5.2.-Los medios como espacio de debate. 6.-Conclusiones.

RESUMEN: En junio de 1983, varios periódicos españoles anunciaron el descubrimiento de un fragmento de cráneo del género Homo, aparecido en Orce, Granada, que parecía revolucionar el panorama científico del momento. Un año más tarde, el mismo fósil fue atribuido a un asno, directamente en el periódico El País. Estalló una gran polémica: el famoso «Hombre de Orce» se transformó en el «Burro de Orce», los descubridores fueron criticados por su precipitación y los científicos se enfrentaron en los medios de comunicación. Mediante un estudio exhaustivo del tratamiento del caso en prensa diaria española, este artículo muestra, en primer lugar, el desarrollo de la controversia y la relación con el contexto histórico que vivía la paleontología y la paleoantropología en Cataluña y España. En segundo lugar, este trabajo también busca entender las razones por las que, en el caso del Hombre de Orce, el consenso entre científicos enfrentados es imposible de alcanzar. Finalmente, el análisis del papel de la prensa en la controversia permite profundizar en la comprensión de la forma en que los medios de comunicación intervienen en los procesos de generación, validación y establecimiento del conocimiento científico.

PALABRAS CLAVE: Paleoantropología, controversia, ciencia y prensa, Hombre de Orce, Institut de Paleontologia de Sabadell, Gibert, de Lumley.

KEY WORDS: Palaeoanthropology, controversy, science and the press, Orce Man, Institut de Paleontologia de Sabadell, Gibert, de Lumley. 
¿Somos alma? ¿Somos materia? ¿Somos sólo fruto del azar? ¿Es fiable el carbono 14? ¿Es nuestro antepasado el hombre de Orce? Siniestro Total, «¿Quiénes somos? ¿De dónde venimos? ¿Adónde vamos?» Julián Hernández. Octubre 1984.

\section{Introducción}

En octubre de 1984, Julián Hernández, líder del grupo musical gallego Siniestro Total, escribió la canción «¿Quiénes somos? ¿De dónde venimos? ¿Adónde vamos?», en la que también se preguntó «¿Es nuestro antepasado el Hombre de Orce?». Este verso es una buena muestra de hasta qué punto, en aquel momento, una polémica científica alrededor de un pequeño fragmento de cráneo estaba en boca de todos. La opinión pública quería saber si el hueso encontrado en Orce era un homínido, uno de nuestros ancestros, o un «simple» asno. Periodistas y científicos dieron su opinión en la prensa escrita, que fue la principal fuente de información para quien quiso seguir el caso.

Este artículo, pretende mostrar la relevancia de la polémica de Orce desde tres puntos de vista, en los que se divide el trabajo. En el primer bloque, una historia cronológica sitúa al Hombre de Orce en la paleontología de la Cataluña de los años ochenta y, especialmente, en relación con el Institut de Paleontologia de Sabadell, institución donde trabajaban sus descubridores. Esta historia cronológica también hace aflorar las interacciones entre los diferentes científicos españoles y entre éstos y los científicos extranjeros. Finalmente, el estamento político, centrado en la Diputació de Barcelona, de la que dependía el Institut, y en la Junta de Andalucía, que otorgaba permisos para excavar en Orce, son también parte importante de la controversia.

En el segundo bloque se enfatizan las particularidades de la paleoantropología, las características anatómicas del fragmento y los mecanismos por los que funcionan las controversias científicas para mostrar cómo en este caso se produce una falta de consenso entre científicos, muy difícil, o imposible, de resolver si ambos «bandos» persisten en sus posiciones.

Por último, este caso es paradigmático para entender cómo los medios de comunicación se transforman en espacios de divulgación, validación y debate científico, ya que éstos estuvieron involucrados en la difusión del 
descubrimiento y en el inicio de la polémica. En el caso de Orce, la prensa se convirtió en un medio que presentó ciencia para el público general, pero también para el público experto.

Para realizar este estudio se han escogido como fuente principal tres periódicos, por ser de gran tirada y representativos de la sociedad española: La Vanguardia, ABC y El País. Otras fuentes utilizadas han sido los artículos científicos, los libros y artículos de divulgación publicados por los investigadores, los documentales emitidos en la televisión y las entrevistas a algunos de los protagonistas ${ }^{1}$.

\section{El Hombre de Orce: «El Hallazgo del Siglo» (o eso parece)}

En el verano de 1976, Josep Gibert, Jordi Agustí y Narcís Sánchez, colaboradores del Institut de Paleontología de Sabadell, descubrieron en la población granadina de Orce el yacimiento de Venta Micena. Durante la campaña de 1982, en la que participaron Gibert, Agustí y Salvador Moyà-Solà, en la dirección científica, se extrajo un fragmento de cráneo que éstos identificaron como un homínido ${ }^{2}$. Los tres científicos eran relativamente jóvenes, no eran profesionales establecidos y ninguno de ellos era experto en homínidos: Gibert tenía 41 años, era experto en insectívoros y trabajaba en un instituto de enseñanza secundaria; Agustí, 28, en roedores y también trabajaba en un instituto; y Moyà-Solà, 27, acababa de leer su tesis sobre bóvidos ${ }^{3}$.

Con el fragmento en la mano, los investigadores fueron a hablar con los políticos de la Diputació de Barcelona, de la que dependía el Institut. Éstos recomendaron enseñarlo a varios expertos nacionales e internacionales. Vieron el fragmento: Domènec Campillo, neurocirujano y paleopatólogo; Miquel Crusafont, paleontólogo que, a pesar de ser ya mayor, era director del

1. Gibert, Josep. El Hombre de Orce, los hominidos que llegaron del sur. Córdoba: Almuzara; 2004. Campillo, Domènec. El cráneo infantil de Orce: el homínido más antiguo de Eurasia. Bellaterra: Edicions Bellaterra; 2002. Martínez, Bienvenido. El Hombre de Orce. Historia de un descubrimiento. Granada: Proyecto Sur; 1993. Pedres que parlen [documental] Guàrdia, Carles; Pou, Francesc. Produccions. Tv3. 1996. 32.49 min, sonido, color. Visualizable en: http:// www.tv3.cat/videos/1390199 [consultado en Jul 2012]. Carandell, Miquel; Hochadel, Oliver. Entrevista a Jordí Agustí 9 Jul 2012; Barcelona. Acosta, Carlos. Entrevista a Josep Gibert 2003. Sabadell, Barcelona.

2. Gibert, n. 1, p. 31.

3. Entrevista Agustí, n. 1. 
Institut y mentor de los descubridores; y los paleontólogos Rafael Adrover, Pierre Mein y Peter Andrews ${ }^{4}$. Todos ratificaron que la pieza pertenecía al género Homo.

A pesar de que la parte interior del fragmento no se podía observar, ya que seguía adherida a una roca, los investigadores creyeron tener suficientes pruebas, y el respaldo de estos expertos, para hacer una publicación del hallazgo $^{5}$. En mayo de 1983, el fragmento, que los investigadores llamaron VM-0, se publicó en la revista Paleontologia i Evolució del Institut de Paleontologia ${ }^{6}$.

En junio, el fósil fue presentado ante la prensa en Granada. Los periodistas que cubrieron la rueda de prensa bautizaron el fragmento como «El Hombre de Orce»: «Se trata, según los expertos, de los restos de uno de los homínidos más antiguos del mundo» ${ }^{7}$.

Según Gibert, Agustí y Moyà-Solà, el Hombre de Orce podría ser el «primer europeo», un homínido que retrasaría la entrada a Europa casi un millón de años ${ }^{8}$. Sugirieron además la llegada de los homínidos por Gibraltar en contra de la comunidad internacional que proponía una llegada por el corredor palestino ${ }^{9}$. El descubrimiento impactó en la prensa y algunos periodistas hablaron de «Revolución científica» $\mathrm{y}$ «Hallazgo del Siglo» ${ }^{10}$. Los

4. Gibert, n. 1, p. 32. Entrevista Agustí, n. 1. Adrover fue un paleontólogo mallorquín, especialista en micromamíferos: Moyà-Solà, Salvador; Pons, Guillem X. Rafel Adrover, paleontòleg, (1911-2007) in memoriam. Bolletí de la Societat d'Història Natural de les Balears, 2007; 49: 213-218. Mein es un paleontólogo francés experto en mamíferos fósiles de la Universidad de Lyon. http://www.icp.cat/index.php/ca/sala-depremsa/noticies-icp/586-honorant-pierremein [consultada Oct 2012]. Peter Andrews fue paleontólogo del Natural History Museum de Londres, especialista en evolución humana, hasta que se retiró en el año 2000. http://www. blandfordtownmuseum.org/research_associates.htm [consultada Oct 2012]. Sobre Crusafont, ver los artículos de Acosta, Florensa y Català en este dossier.

5. Gibert, n. 1, p. 33.

6. Gibert, Josep; Agustí, Jordi; Moyà-Solà, Salvador. Presencia de Homo sp. en el yacimiento del Pleistoceno inferior de Venta Micena (Orce, Granada). Paleontologia i Evolucio. 1983; Publicación Especial 1-9.

7. De Semir, Vladimir. Tres paleontólogos catalanes descubren el hombre más antiguo de Europa y Asia. La Vanguardia. 12 Jun 1983: 51.

8. En aquel momento los homínidos más antiguos de Europa, la mandíbula de Mauer y el cráneo de Tautavel, tenían algo más de 0'5 millones de años. Los descubridores databan el fragmento de Orce entre 0'9 y 1'6 millones de años. Dennell, Robin; Roebroeks, Wil. The earliest colonization of Europe: the short chronology revisited. Antiquity. 1996; 70: 535-42. Gibert, n. 1, p. 273-294.

9. Agustí, Jordi; Gibert, Josep; Moyà-Solà, Salvador, Características del Hombre de Orce. La Vanguardia. 21 Ago 1983: 26.

10. Aguilar, José. El hallazgo del Hombre de Orce puede suponer una revolución en el estudio de la especie humana. El País. 14 Jun 1983, y Lyon, W. Huesos Extraños. El País. 15 Jun 1983. 
descubridores escribieron sus propios artículos en periódicos y revistas de divulgación, dieron varias conferencias e incluso planearon escribir un libro de divulgación científica ${ }^{11}$. Durante la excavación de 1983, los reconocidos científicos franceses Marie-Antoinette y Henry de Lumley visitaron Orce, confirmaron que VM-0 era un homínido e invitaron a Gibert, Agustí y Moyà-Solà a estudiar el fragmento en Francia ${ }^{12}$.

Ese mismo verano, Crusafont murió y Gibert, ya famoso por «su» Hombre de Orce fue nombrado, por los dirigentes de la Diputació, director en funciones del rebautizado, Institut de Paleontologia Miquel Crusafont de Sabadell ${ }^{13}$.

De octubre de 1983 a abril de 1984, durante la limpieza de la parte interna del fragmento, apareció una pequeña cresta que hizo dudar a los investigadores. Gibert, Agustí y Moyà-Solà llevaron el fragmento a París para que lo viera el matrimonio de Lumley. Cuando volvió a examinar el fragmento, Marie-Antoinette se convenció de que no se trataba de un homínido sino de un miembro juvenil del género Equus, concretamente un asno. En este punto, Agustí y Moyà-Solà aceptaron la opinión de la francesa pero Gibert no lo hizo. El grupo de científicos descubridores se rompió ${ }^{14}$.

El 12 mayo de 1984, cuando los descubridores volvían de París, El País publicó en portada las dudas alrededor del Hombre de Orce después de una llamada de la misma Marie-Antoinette de Lumley ${ }^{15}$ (Figura 1).

Díaz-Rojo ha analizado la retórica de los medios en las primeras noticias del descubrimiento: Díaz Rojo, José Antonio. Retórica científica en la prensa. El hallazgo paleontológico del cráneo de Orce (1983). In: Díaz Rojo, José Antonio, ed. La circulación del saber científico en los siglos XIX y XX. Valencia: Editorial de la Universidad de Valencia; 2011, p. 99-128.

11. Un ejempo de conferencia: Día a Día. La Vanguardia. 22 Feb 1984: 53. Para una revista de divulgación: Agustí, Jordi; Gibert, Josep; Moyà-Solà, Salvador. El Hombre de Orce. Revista de Arqueología. 1983; 29: 16-21. El libro fue anunciado en Piñol, Rosa Maria. La nova editorial catalana Empúries publicará diveres obres de Riba. La Vanguardia. 15 Mar 1984.

12. Los de Lumley, que trabajaban en varios yacimientos de todo el mundo, eran famosos por ser los descubridores del Hombre de Tautavel. Henry era director del Institut de Paléontologie Humaine y del laboratorio de prehistoria del Musée de l'Homme ambos en París. http:// fr.wikipedia.org/wiki/Henry_de_Lumley [consultada Oct. 2012] Gibert, n. 1, p. 40. Sobre de Lumley puede verse también el artículo de Hochadel en este dossier.

13. Gibert, n. 1, p. 40-41. Entrevista Agustí, n. 1.

14. Entrevista Agustí, n. 1.

15. Relaño, Alfredo. Serios indicios de que el cráneo del «hombre de Orce» pertenece a un asno. El País. 12 May 1984: 1. Relaño, Alfredo. Serias sospechas de que el «hombre de Orce» no es un hombre, sino que podría ser un asno de dos a cuatro meses de edad. El País. 12 May 1984: 22. 


\section{Dudas sobre la validez de un hallazgo histórico}

En junio de 1983, en la localidad granadina de | silizado hizo pensar que se trataba de un hombre | sobre el origen humano de esos restos y han susOrce, paleontólogos andaluces y catalanes anun- de una antigledad entre 900.000 y 1.600 .000 pendido un simposio en el que se iba a estudiar el ciaron lo que podria ser uno de los descubrimien- antos, lo que le convertía en el resto humano más hallazgo. Las últimas investigaciones hacen sostos paleontológicos más importantes de los últi- viejo encontrado en Eurasia. Casí un año des- pechar que, en realidad, el Hombre de Orce po-

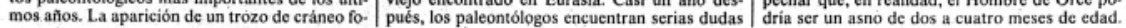

\section{Serias sospechas de que el 'hombre de Orce' no es un hombre, sino que podría ser un asno de dos a cuatro meses de edad}
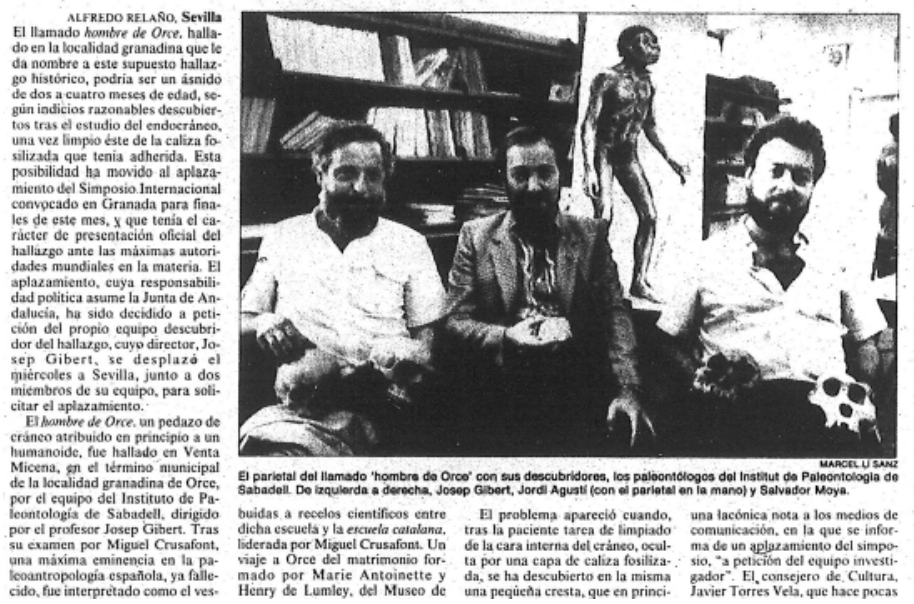

recorrido Paris-Niza-PerpinainMadrid. Seguin nuestras informasa Maric Antoinetre de Lumiey quien aconsejo a Josep Gibert la suspensión del simóosio, e incluso llegó a decirle que si no se realiza: ba tal suspenswin ella y su marido pretextarian una enfermedad para Marie Antoincite de Lumley re. cibió del equipo de Gibér un molde del endoctance del hombre de Oren y fue ella quien, tras un estu. dro de su cyuipo, recontendo comcuatro meses de edad De hecho. el equipo de Gibert ha recopilado en las ultimas fechas crineos de asnos jovenes a fin de estudiar las suturas interiores de los huesos. El profesor Gibert se enconira. ba ayer en Paris. estudiando con el matrumono Lumley, fuertemente comprometido tamien cen el ha. llazgo. las posibles salidas a la cuestion. Asidro foro, unico muem. rídico pudo entrar en conte pe. ayer, admitio que existia un zio de posibilidades de que no se tra tara de un hombre. pero expres su esperanza en la falta de estuh Jies sobre endocraneos de esa antiguedad $y$ en el hecho de que se pucjera descartar firnemente que quier tipo decarni-

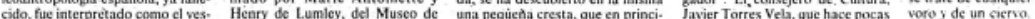

Figura 1. Artículo en El País que anunció las dudas sobre el fragmento de Orce. Relaño, Alfredo. Serias sospechas de que el «hombre de Orce» no es un hombre, sino que podría ser un asno de dos a cuatro meses de edad. El País. 12 May 1984: 22.

La polémica aterrizó en los medios de comunicación. El seguimiento fue espectacular, en solo cuatro días El País llegó a publicar hasta once artículos sobre la controversia ${ }^{16}$. Aparecieron artículos de opinión, se preguntó a otros científicos e incluso los caricaturistas tomaron la palabra $^{17}$ (Figura 2).

16. Por ejemplo: Redacción. Un gran descubrimiento presentado sin reservas. El País. 12 May 1984: 23. Redacción. El descubridor del «hombre de Orce» insiste en su autenticidad. El País. 13 May 1984. Relaño, Alfredo. El pueblo granadino de Orce, indiferente ante la polémica sobre el origen del cráneo encontrado en Venta Micena. El País. 14 May 1984. Navarro Arisa, J.J. El director del Museo del Hombre de París no descarta que el fósil de Orce sea humano. El País. 15 May 1984.

17. Por ejemplo: Redacción. Hombre o borrico. El País. 15 May 1984. El Misterio del Hombre de Orce. El Papus. 525, Jun 1984. 


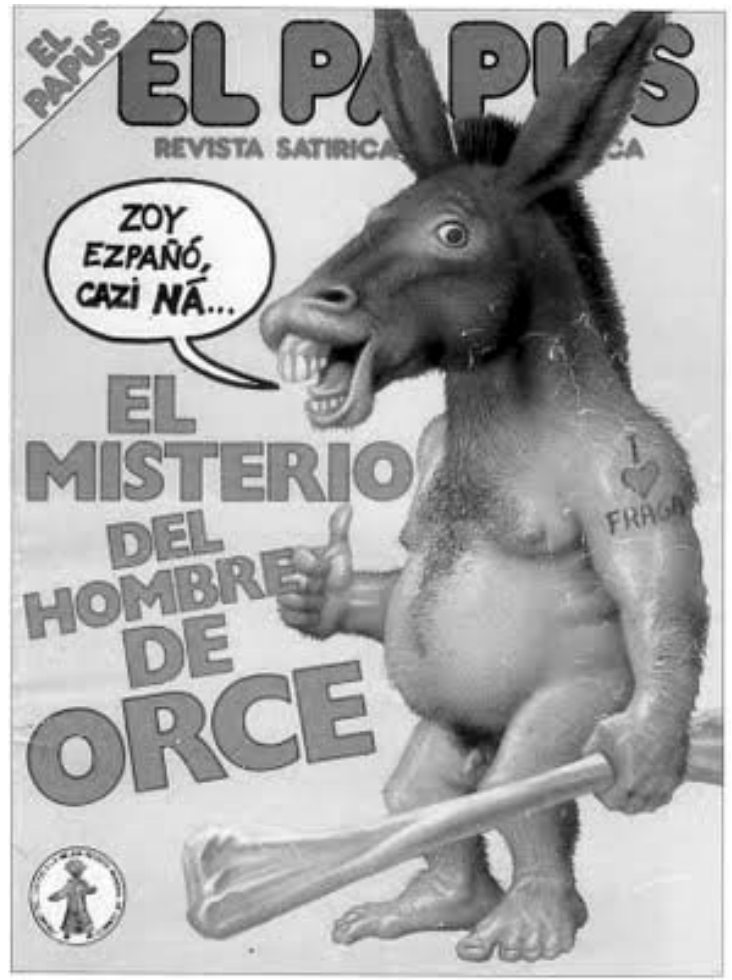

Figura 2. Portada de la revista satírica El Papus de junio de 1984 en plena polémica pública. Fuente: Díaz Rojo, José Antonio. Paleoantropología y prensa. El tratamiento periodístico del «hombre de Orce». Mètode. Revista de difusión de la investigación de la Universitat de València, 53: 37.

La forma en que estalló la controversia visibiliza las dos caras de la relación entre los científicos catalanes, conscientes de que procedían de un país «retrasado» en ciencia y los científicos franceses, de gran prestigio $^{18}$.

18. Agustí afirma «Nos pusimos en manos de de Lumley, en aquella época era Monsieur L'Empereur»; Entrevista Agustí, n. 1. En las primeras crónicas del hallazgo los descubridores se identifican como catalanes y herederos de Crusafont y el Institut de Paleontologia de Sabadell. Agustí, Jordi; Gibert, Josep; Moyà-Solà, Salvador. La investigación paleontológica. La Vanguardia. 21 Ago 1983: 26. González, Evaristo. El Hombre de Orce sigue asombrando. La Vanguardia. 11 Mar 1984: 49. 
Por un lado, los descubridores fueron a París en busca de la opinión de los «expertos» franceses y, por el otro, el rechazo de Gibert a aceptar esta opinión «experta», y su posterior argumentación sobre este rechazo, se enmarcaron dentro de un discurso de superación de un supuesto «colonialismo» científico en la paleontología española ${ }^{19}$.

En mayo de 1985, tres catedráticos de paleontología, y también antiguos discípulos de Crusafont, Miquel de Renzi, Jaume de Porta y Jaume Truyols, enviaron una carta a todos los periódicos en la que criticaron la «difusión imprudente» que se hizo del descubrimiento e insinuaron que Gibert no «contrastaba» sus «hipótesis», concluyendo que para ellos «esa era la diferencia entre un paleontólogo y un mero coleccionista de fósiles» ${ }^{20}$. Según Gibert, estas afirmaciones hicieron un daño «terrible» a su persona y a su prestigio como científico, llegando a afirmar que esta carta podría estar relacionada con su cese como director del Institut, que se produjo en octubre de ese año ${ }^{21}$. Jordi Agustí ocupó el lugar de Gibert, que mantuvo una posición de investigador ${ }^{22}$.

En el verano de 1986, la Junta de Andalucía negó el permiso de excavación a Gibert. En los siguientes 21 años de controversia, de 1986 a 2007, aunque excavó en otros yacimientos cercanos, Gibert sólo pudo excavar en Venta Micena cuatro veranos. El mismo Gibert afirmó: «mi lucha anual por un papel, un permiso para trabajar, ha sido, con mucho, peor que la polémica científica» ${ }^{23}$.

Ante estas dificultades, Gibert y su equipo, del que ya no formaban parte Agustí y Moyà-Solà, se centraron en demostrar que VM-0 era un homínido. Este equipo estaba formado, o contaba con la colaboración, entre otros, de Domènec Campillo, que analizaba las características anatómicas del fragmento; Paul Palmqvist, biólogo matemático que publicó con Gibert; Bienvenido Martínez, doctorado de Gibert, experto en fauna

19. Gibert, n. 1, p. 52. Más sobre este «colonialismo» en Hochadel, Oliver. El mito de Atapuerca. Orígenes, ciencia, divulgación. Bellaterra: Edicions UAB; 2013, p. 35-72.

20. De Renzi, Miquel; Porta, Jaume; Truyols, Jaume. Nota sobre paleontologia. Avui. 6 Mar 1985: 10.

21. Gibert, n. 1, p. 56-57. Un «accidente» con la Guardia Civil respecto a unos permisos de excavación en Cueva Victoria, un yacimiento en Murcia que también conducía Gibert, también parece estar relacionado con esta destitución. Jover, Jaime. La Guardia Civil desaloja de Cueva Victoria a 7 arqueólogos catalanes. La Vanguardia. 11 Oct 1985: 35.

22. Gibert, n. 1, p. 56. Gibert ocupó la dirección durante poco más de dos años, de agosto de 1983 a octubre de 1985. Entrevista Agustí, n. 1.

23. Gibert, n. 1, p. 105. 
y director del Museo de Orce; Lluís Gibert, geólogo e hijo de Josep; y Enrique García-Olivares, que desde la Universidad de Granada realizaba análisis inmunológicos del fragmento ${ }^{24}$. La investigación transcurrió por diversas vías, desde estas innovadoras pruebas inmunológicas a pruebas de fractales o comparaciones anatómicas ${ }^{25}$. Además de un gran número de publicaciones surgidas de estas investigaciones, Bienvenido Martínez, escribió en 1993 un pequeño libro de divulgación sobre la historia de las excavaciones y Gibert, en 2004, también escribió un libro con su historia y teorías científicas ${ }^{26}$. Gibert esgrimió a menudo todas estas publicaciones para legitimar y reforzar su posición en la controversia: «[Gibert y sus colaboradores han publicado] 138 trabajos (12 de ellos en revistas de impacto y 15 en revistas internacionales) [...] Esta es la base real del progreso científico» ${ }^{27}$. Con esta estrategia de publicación intensiva, Gibert trató, además de contestar a todos sus oponentes y críticos, de hacer circular y aumentar la credibilidad de sus ideas ${ }^{28}$.

En septiembre de 1986, Gibert acudió al recién creado congreso mundial de Arqueología en Southampton ${ }^{29}$. La prensa consideró que este congreso, que no era exclusivamente de especialistas en paleoantropología, era el lugar donde la comunidad científica internacional confirmaría si el fragmento de Orce era humano o no: «la verdad sobre el origen humano del cráneo se expondrá y será ratificada en la reunión arqueológica mundial» ${ }^{30}$.

24. Los análisis de García-Olivares, pioneros en Europa, buscaron, con resultado positivo, albúmina humana en el fragmento de Orce y en otros supuestos restos homínidos. Ver, por ejemplo: Borja, Concepción; García-Pacheco, Marcos; García-Olivares, Enrique; Scheuenstuhl, G.; Lowenstein, J. Immunospecificity of albumin detected in 1.6 million-year-old fossils from Venta Micena in Orce, Granada, Spain. American Journal of Physical Anthropology. 1997; 103: 433-441.

25. Las «dimensiones fractales», establecidas mediante complicadas fórmulas matemáticas, sirven en este caso para establecer la complejidad de las suturas. Cuanto más sencillas más probabilidad de situar el fragmento dentro del género Homo. Gibert, Josep; Palmqvist, Paul. Fractal analysis of the Orce skull sutures. Journal of Human Evolution. 1995; 28: 561-575.

26. Gibert, n. 1 y Martínez, n. 1.

27. Antes de la controversia, de 1975 a 1983, Gibert había publicado una veintena de artículos científicos, sobre todo en las revistas Paleontologia i Evolució y Acta Geológica Hispania, basados principalmente en su tesis doctoral en insectívoros españoles. http://www.fundacionjosepgibert.org/node/3 [Consultada Oct. 2012]. La cita en: Gibert, n. 1, p. 406.

28. Para un ejemplo similar: De Bont, Raf. The creation of Prehistoric man: Aimé Rutot and the Eolith controversy, 1900-1920. Isis. 2003; 94 (4): 604-630 (607).

29. Golson, Jack. The World Archaeological Congress, Southampton, and its aftermath. Australian Archaeology. 1986; 23: 100-105 (100).

30. Paredes, José Antonio. Gibert: las dudas sobre el «hombre de Orce» acabarán en Southampton. La Vanguardia. 23 Ago 1986: 20. 
Después del congreso, donde nadie pareció poner objeciones a Gibert, el El País tituló «El cráneo de Orce es humano, según confirma el Congreso Arqueológico Mundial» ${ }^{31}$.

El año siguiente, en octubre de 1987, Gibert asistió a otro congreso en Turín. En una sesión previa, Marie-Antoinette de Lumley criticó el Hombre de Orce sin la presencia de Gibert. Cuando éste expuso sus argumentos, de Lumley no puso objeción alguna, por lo que el debate científico, para Gibert absolutamente necesario, no se produjo ${ }^{32}$. Con Gibert aún en Turín, sus antiguos colaboradores, Agustí y Moyà-Solà, anunciaron en la prensa española la publicación de un artículo científico en el que expondrían su cambio de opinión respecto a VM- $0^{33}$. Éste fue el primer artículo científico que defendió que el fragmento pertenecía al género Equus ${ }^{34}$.

\section{Congreso Internacional de Paleontología Humana en Orce: «Josep Gibert tenía razón» (o eso parece)}

Después de unos años de calma mediática, en septiembre de 1995, Gibert organizó un Congreso Internacional de Paleontología Humana en Orce, que tuvo un seguimiento casi diario en la prensa ${ }^{35}$. Gibert y su equipo presentaron los estudios realizados hasta el momento alrededor de VM-0 y los yacimientos en Orce $^{36}$. Según la prensa, los asistentes al congreso parecieron aceptar la presencia de homínidos en la zona ${ }^{37}$. Al final del con-

31. Moreno, Manuel. El cráneo de Orce es humano, según confirma el Congreso Arqueológico Mundial. El País. 9 Sep 1986.

32. Gibert, n. 1, p. 78. Acosta, Entrevista Gibert, n. 1.

33. Mercadé, Josep. Los colaboradores de Gibert dicen que el cráneo de Orce pertenece a un equino. La Vanguardia. 2 Oct 1987: 40. Redacción. Dos investigadores del cráneo de Orce afirman que perteneció a un équido joven. El País. 2 Oct 1987: 40.

34. Agustí, Jordi; Moyà-Solà, Salvador. Sobre la identidad del fragmento craneal atribuido a Homo sp. de Venta Micena (Orce Granada). Estudios Geológicos. 1987; 42: 538-443.

35. Por ejemplo: De Leon Sotelo, Trinidad. José Gibert: «El Congreso de Orce cambiará la prehistoria en Europa». ABC. 2 Sep 1995: 53. Un congreso internacional asegura que el hombre de Orce es el europeo más antiguo. La Vanguardia. 5 Sep 1995: 36. Incluso tuvo cierta repercusión internacional: Denison, Simon. 1.8 million-year-old human presence claimed in Spain. British Archaeology. 1995: 7. Weimer, Wolfram. Wo lebte der erste Europäer? Frankfurter Allgemeine Zeitung. 7 Set 1995: 12.

36. Gibert, n. 1, p. 91.

37. Por ejemplo: Turbón, Daniel. Hombre de Orce: Josep Gibert tenía razón. La Vanguardia. 17 Sep 1995: 64. 
greso, la Junta de Andalucía prometió permisos de excavación y recursos económicos. Diego Valderas, Presidente del Parlamento andaluz y presente en el congreso, subrayó que Gibert había «ganado la batalla de la opinión pública y la de la cámara andaluza» ${ }^{38}$.

Esta «victoria pública» no duró demasiado. El mes siguiente, José María Bermúdez de Castro y Eudald Carbonell, dos miembros del triunvirato de directores de Atapuerca, hicieron unas declaraciones en un artículo titulado «Paleontólogos y geólogos cuestionan la existencia del "hombre de Orce"». Bermúdez afirmó que: «a partir de los fósiles presentados, el hombre de Orce no existe», mientras que Carbonell mantuvo que: «las afirmaciones que se han hecho [en Orce] no se ajustan a los convenios de rigor científico internacional» ${ }^{39}$.

Durante la polémica, Gibert mantuvo un «aura» de científico excéntrico y poco ortodoxo, y acarreó un «estigma» que le llevó a «luchar» por dinero y permisos, recibiendo críticas de otros científicos y periodistas ${ }^{40}$.

Además, Gibert se caracterizaba a si mismo como un científico al margen y revolucionario. En su libro de divulgación, Gibert cita el libro de Thomas S. Kuhn, La estructura de las revoluciones científicas, para ejemplificar que «su» Hombre de Orce supone un «nuevo paradigma» y el hecho de que no sea aceptado simplemente responde a que los «cambios de paradigma» necesitan tiempo ${ }^{41}$. En mi opinión, usando a Kuhn, describirse como un marginado-revolucionario incluso sale a cuenta. Desde esta posición sólo puedes ganar adeptos hasta que tus ideas sean aceptadas. Eso demostrará que tenías la razón desde el inicio y que los opositores eran científicos anclados en el pasado ${ }^{42}$.

Después del aparente triunfo del congreso de Orce, el equipo de Gibert se volvió a romper. El verano siguiente, tres miembros de éste: Martínez, Palmqvist y Alain Turq (arqueólogo francés que había excavado

38. La Consejería promete apoyo económico para el yacimiento de Orce. ABC. 9 Sep 1995: 39.

39. Rivera, Alícia. Paleontólogos y geólogos cuestionan la existencia del «hombre de Orce». El País. 6 Oct 1995.

40. Pedres que parlen, n. 1, documental emitido sobre estas mismas fechas, describe al grupo de Gibert como un grupo que trabaja en «la clandestinidad científica». Sella, Antoni. Paleontólogos a la Greña. La Vanguardia. 3 Feb. 1996: 10-13, afirma que Gibert «presta más atención a su intuición que al rigor científico», p. 12.

41. Gibert, n. 1, p. 56.

42. Fahnestock describe una situación similar en la controversia «Pre-Clovis». Fahnestock, Jeanne. Arguing in different forums: The Bering crossover controversy. Science, Technology \& Human Values. 1989; 14 (1): 26-42 (29). 
las industrias líticas de Fuente Nueva 3, un yacimiento cercano a Venta Micena), anunciaron, primero en el periódico local de Orce, La Alcazaba, y más tarde en la televisión autonómica andaluza, Canal Sur, que, cansados de la falta de permisos y financiación, decidían escindirse del equipo. Además, afirmaron que VM-0 era un burro y formaron un nuevo equipo para excavar en Orce, junto con antiguos colaboradores de Gibert como Agustí o Isidro Toro, un arqueólogo granadino que ya había trabajado en Venta Micena ${ }^{43}$.

Una semana después de las declaraciones en Canal Sur, José Ramón Martínez Olivares, teniente de alcalde de Orce, destituyó a Bienvenido Martínez como director del Museo de Orce ${ }^{44}$. Este cese pone de manifiesto la conveniencia, para el pueblo de Orce, de que el Hombre de Orce fuera aceptado, ya que eso haría del fragmento una atracción turística ${ }^{45}$. La escisión volvió a llevar la controversia a los periódicos. La Junta volvió a negar los permisos de excavación y las subvenciones prometidas durante el congreso ${ }^{46}$.

Este ejemplo muestra como la polémica científica en los medios tuvo repercusión en los políticos que, a su vez, repercutieron en las investigaciones científicas. El hecho de que, a raíz del congreso de Orce, se prometieran ayudas que fueron de nuevo negadas con el retorno de la polémica, muestra como el estamento político también fue un público que recibió, y reaccionó, ante el impacto en los medios. Los políticos fueron entonces otro «actor» en la controversia científica del Hombre de Orce.

Además, las declaraciones de Palmqvist, Martínez y Turq, primero en la publicación local y después a la televisión andaluza, y la expulsión de Martínez del Museo, muestran el «poder» que Gibert mantenía en Orce, donde iba cada verano, con permiso de excavación o no, con toda su familia. Gibert fue nombrado hijo adoptivo del pueblo y el Museo de Orce se rebautizó como Museo «Josep Gibert» ${ }^{47}$. Así, Gibert fue marginado dentro de la comunidad científica, que lo tachó de «poco científico» pero, a la vez,

43. De León Sotelo, Trinidad. El equipo del «hombre de Orce» va de cráneo. ABC. 4 Ago 1996: 49.

44. De León Sotelo, Trinidad. El alcalde de Orce expulsa al científico que no cree que el fragmento craneal es de un homínido. ABC. 23 Ago 1996: 33.

45. En la prensa aparece la voluntad de montar un parque arqueológico en Venta Micena: La Junta de Andalucía deniega el permiso para excavar Orce «a la espera de un plan». ABC. 20 Ago 1994: 45.

46. De Leon Sotelo, Trinidad. Orce, una polémica que no fosiliza. ABC. 8 Ago 1996: 47.

47. Gibert, n. 1, p. 425. 
en el pequeño pueblo de Orce, que a principios de los ochenta contaba con menos de 2000 habitantes, siguió siendo un personaje reconocido y con prestigio, y de ahí el intento de los «disidentes» de «ensuciar» su imagen en medios locales ${ }^{48}$.

A partir de aquí, la polémica se diluyó. En 1999, la Junta decidió crear una comisión internacional que coordinaría unas excavaciones dirigidas a tres bandas, por Gibert, Toro y Agustí. Estas excavaciones se realizarían con miembros de los dos grupos. Ahora bien, y según Gibert, poco a poco, su grupo fue apartado de las investigaciones. En 2003 se publicó una monografía firmada por Toro, Agustí y Martínez, que presentó los resultados de las campañas supervisadas por la comisión desde $1999^{49}$.

En 2006, Campillo publicó el descubrimiento de un cráneo de una niña de época romana que presentaba la misma cresta polémica que el fragmento de Orce ${ }^{50}$. Se organizó una rueda de prensa y algunos periódicos afirmaron que ésta era la prueba definitiva y que Gibert «Cantó victoria» ${ }^{51}$. Pero la llamada comunidad científica hizo caso omiso de esta publicación.

En octubre de 2007, Josep Gibert i Clos murió víctima de un cáncer linfático. Sus cenizas fueron esparcidas por el yacimiento de Venta Micena. El Hombre de Orce siguió, y sigue, apareciendo en periódicos y revistas de divulgación ${ }^{52}$. Hallazgos recientes en Orce y Atapuerca parecen haber confirmado la presencia, hace más de 1 millón de años, de homínidos en la Península ${ }^{53}$.

\footnotetext{
48. Habitantes Orce: http://es.wikipedia.org/wiki/Orce [consultada Julio 2012].

49. Toro, Isidro; Agustí, Jordi; Martínez, Bienvenido. El Pleistoceno Inferior de Barranco León y Fuente Nueva 3, Orce (Granada). Campañas 1999-2002. Sevilla: Junta de Andalucía; 2003.

50. Campillo, Domènec; Milagros, María; García, Elena. An occipital crest in an infant cranium from the Roman necropolis of Francolí (Tarragona, Spain): implications to the interpretation of the Orce skull. Revista Española de Antropología Física. 2006; 26: 93-102.

51. Planas, Mónica, Un hombre marcado por un fósil. La Vanguardia. 9 Oct 2007: 37.

52. Por ejemplo: Cardeñosa, Bruno. El Hombre de Orce el primer habitante de la península. Historia de iberia vieja. 2007; 29: 78-81, o Campillo. El Hombre de Orce es un homínido. Revista Arqueología. 2010; 35: 14-21.

53. Vida humana en Orce hace 1,3 millones de años. El País. $21 \mathrm{Sep} 2010$. Carbonell, Eudald et al. The first hominid of Europe. Nature. 2008; 452: 465-469.
} 


\section{Ocho centímetros de polémica: VM-0 y la imposibilidad del consenso científico}

VM-0 es un fragmento de cráneo, dos porciones de los huesos parietales y una pequeña parte del occipital, que cabe en la palma de la mano. El Hombre de Orce no tiene más de 8 centímetros de diámetro. Sacar de él información útil es una tarea difícil (Figura 3).

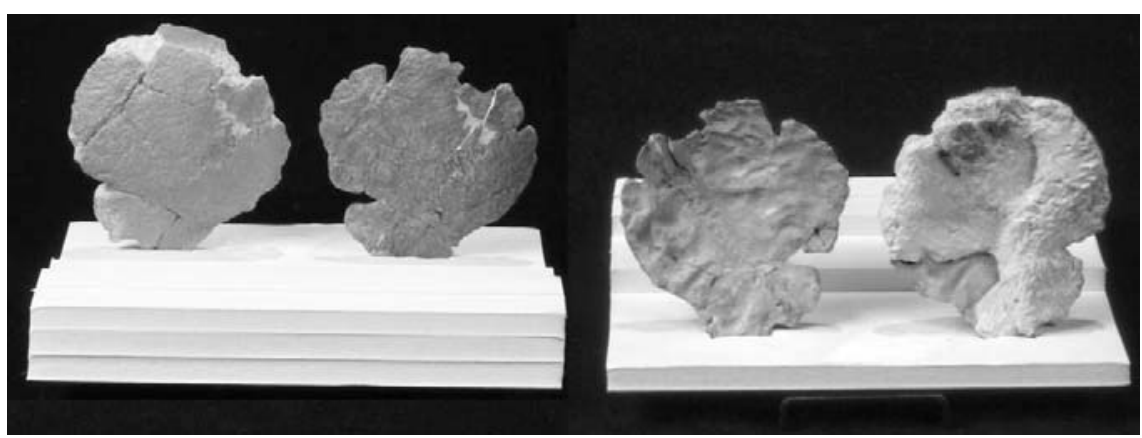

Figura 3. Moldes del fragmento VM-0, el Hombre de Orce. Izquierda: parte externa antes y después de la limpieza. Derecha: parte interna, antes y después de la limpieza.

Fuente. http://hicido.uv.es/Expo_medicina/Morfologia_XIX/evolucion.html. [Consultada Oct. 2012]

En el momento de la primera publicación, el fragmento, que aún tenía la parte interna cubierta de roca calcárea, presentaba, para los investigadores, suficientes características para atribuirlo al género Homo: una curvatura transversal y longitudinal amplia (característica que indica un cráneo de gran tamaño), poco grosor (que presentan los individuos infantiles de Нотo) y ausencia de sutura coronal en la parte exterior (sutura típica de mamíferos no humanos $)^{54}$. Después de la limpieza, apareció una cresta occipital que hizo dudar a los investigadores. De Lumley hizo estallar la polémica afirmando que se trataba de un espécimen del género Equus.

Analizaremos aquí las características que se presentaron para clasificar el fragmento y las opiniones de los investigadores: 
- Pequeña cresta interna en el occipital: es la cresta que causó la polémica. Para los defensores de Equus, es la norma en el grupo y, en cambio, en Homo es una excepción ${ }^{55}$. Para el otro bando, esta cresta entra en la variabilidad de Homo, que es muy grande para el occipital $^{56}$. Gibert detecta la cresta en un fósil africano de Homo ergaster y Campillo en una niña de época romana ${ }^{57}$.

- Sutura coronal en el endocráneo: Para los contrarios al Hombre de Orce, VM-0 muestra una sutura coronal en la parte interior, propia del género Equus. Esta sutura no se observa en la cara exterior porque ha sufrido abrasión ${ }^{58}$. Por el contrario, según Gibert y Campillo, esta sutura no existe y lo que ven los otros investigadores es una fractura póstuma ${ }^{59}$.

- Impresiones digitales: la cara interna presenta unas pequeñas pero profundas impresiones digitales ${ }^{60}$. Según los partidarios de Equus, Homo «habitualmente» no presenta impresiones, en cambio los equinos sí ${ }^{61}$. Para Campillo, las impresiones digitales son normales en un cráneo en formación, como se suponía que era el de Orce ${ }^{62}$.

- Curvatura transversal y longitudinal: VM-0 tiene una curvatura que hace pensar en un cráneo muy amplio, típico de los homíndos ${ }^{63}$. Los detractores de Homo admiten este hecho pero argumentan que una curvatura amplia se puede dar en caballos y rinocerontes jóvenes ${ }^{64}$. Agustí, por su parte, afirma que esta curvatura es consecuencia de una modificación por el peso del sedimento ${ }^{65}$.

55. Agustí; Moyà-Solà, n. 34, p. 537.

56. Gibert, Josep et al. Caracteristicas diferenciales entre el fragmento de craneo de Homo sp. de Venta Micena (Orce, Granada) y los Equidos. Estudios Geológicos. 1989; 45: 121-138 (127).

57. Martínez, n. 1, p. 27-28. Campillo; Milagros; García, n. 50.

58. Agustí; Moyà-Solà, n. 34, p. 538. Moyà-Solà, Salvador; Köhler, Meike. The Orce skull. Anatomy of a mistake. Journal of Human Evolution. 1997; 33: 91-97 (96).

59. Gibert, Josep; Campillo, Doménec; García-Olivares, Enrique. Hominid status of the Orce cranial fragment reasserted. Journal of Human Evolution. 1998; 34: 203-217 (212).

60. Se llama impresiones digitales a las depresiones en el interior del cráneo que corresponden a los surcos y giros del cerebro. Estas impresiones quedan marcadas cuando cráneo esta en formación. Liem, Torsten. La Osteopatia Craneosacra. Barcelona: Paidotribo; 2002, p. 53.

61. Moyà-Solà; Köhler, n. 58, p. 94-95.

62. Campillo, n. 1, p. 172.

63. Campillo, n. 1, p. 174.

64. Moyà-Solà; Köhler, n. 58, p. 92.

65. Entrevista Agustí, n. 1. 
En definitiva, hablando de los mismos caracteres, los investigadores llegan a conclusiones diferentes y raramente hacen concesiones a las otras interpretaciones.

Habitualmente, los paleoantropólogos encuentran restos muy fragmentarios, pero, a pesar de esto, los interpretan como grandes hallazgos, restando importancia a fósiles anteriores. Así, a menudo, los diferentes intereses entran en conflicto provocando arduas polémicas científicas ${ }^{66}$.

En el caso de Orce, esta falta de consenso nos lleva a considerar la ausencia de árbitros en el proceso. Como Elisabeth S. Clemens ejemplifica, citando a Pierre Bourdieu, en su artículo sobre el descubrimiento del asteroide que provocó la extinción de los dinosaurios, cuando los criterios con que se juzga un determinado hecho son también parte de la controversia, no puede haber jueces, ya que no hay ningún juez que no sea parte también de la controversia ${ }^{67}$.

Por otro lado, en aquel momento no había prácticamente otros especímenes de cráneos de homínidos de esa antigüedad para comparar con el Hombre de Orce. Esto nos lleva a aplicar la idea del «Experimenters' regress» o en este caso el «Paleoanthropologists' regress» ${ }^{68}$. Si la clasificación del Hombre de Orce como Homo depende de si presenta, o no, la sutura coronal o de si la cresta occipital es propia de los homínidos y no hay especímenes para determinar si los homínidos de esa época tenían, o no, la cresta y la sutura, entonces, no hay posibilidad, basándose en estos caracteres, de determinar si el Hombre de Orce es un homínido o no. Así, si hay opiniones divergentes y tampoco hay jueces que arbitren el proceso, no puede haber consenso ya que la clasificación definitiva es imposible, siendo así la controversia imposible de solucionar.

66. Pelayo, Francisco. Controversias científicas y repercusiones sociales de la paleontología humana. Mètode. 2008; 53: 22-24. Lewin, Roger. Bones of contention. Chicago: The University of Chicago Press; 1987.

67. «As the definition of the criteria of judgment [...] is itself in a struggle, there are no judges, because there is no judge who is not also a party to the dispute». Clemens, Elisabeth. Of asteroids and dinosaurs. The role of the press in the shaping of scientific debate. Social Studies of Science, 1986; 16: 421-456 (425).

68. Como hemos visto, Gibert sólo identifica uno de Homo Ergaster. Martínez, n. 1, p. 27-28. Collins, Harry. Changing order: Replication and induction in scientific practice. Chicago: The University of Chicago Press, 1985. Maerker, Anna. The tale of the hermaphrodite monkey: classification, state interests and natural historical expertise between museum and court, 1791-1794. The British Journal for the History of Science. 2006; 39: 29-47. 


\section{5. «Una dimensión distinta»: los medios de comunicación y el Hombre de Orce}

\subsection{Divulgación y validación en la prensa}

Poco después de la presentación del hallazgo, Gibert, Agustí y Moyà-Solà publicaron en La Vanguardia varios artículos donde describieron las características de VM-0, su descubrimiento, su (enorme) importancia dentro del mapa de la paleoantropología mundial y las precarias condiciones de financiación en las que trabajaban cuando lo encontraron ${ }^{69}$. Varios años de controversia más tarde, en 1993, Gibert publicó, también en La Vanguardia, un artículo a doble página describiendo las pruebas aportadas hasta el momento en favor del Hombre de Orce. Gibert decía que los progresos realizados durante los últimos años se debían a la gran capacidad de trabajo de su equipo (y no a las escasas subvenciones recibidas) ${ }^{70}$.

En estos ejemplos vemos como los científicos dan a conocer sus investigaciones en público para recibir apoyo institucional, popular y periodístico. Como afirma Greg Myers, saber explicar las propias investigaciones a la sociedad es esencial para conseguir dinero ${ }^{71}$. Además, Gibert, buscaba limpiar, y controlar su propia imagen, deteriorada tras años de polémica ${ }^{72}$.

En octubre de 1987, Moyà-Solà y Agustí difundieron en la prensa la publicación de un artículo en la revista científica Estudios Geológicos en el que defendían que el Hombre de Orce era un miembro del genero Equus. El anuncio de una novedad científica aún no publicada es una práctica que ha sido llamada science per press conference y que puede tener varios

\footnotetext{
69. Agustí; Gibert; Moyà-Solà, n. 9.

70. Gibert. El primer ser humano de Europa. La Vanguardia. 1 May 1993: 10-11.

71. «Being able to explain one's project, and its relevance to wider society, [...] is an essential part of running a large lab and getting further funding». Myers, Greg. Discourse studies of scientific popularisation: questioning the boundaries. Discourse Studies. 2003; 5 (2): 265-279 (270). Otros ejemplos de la financiación en paleoantropologia en: Kjærgaard, Peter. The fossil trade: Paying a price for human origins. Isis. 2012; 103 (2): 340-355.

72. Como afirma Hochadel: «Writing the history of one's own research is a way to control it» Hochadel, Oliver. Atapuerca - the making of a magic mountain. Popular science books and human-origins-research in contemporary Spain. In: Schirrmacher, Arne, ed. Communicating Science in 20th Century Europe. A Survey on Research and Comparative Perspectives. Prepint Max-Planck-Institute for the History of Science. 385, 2009; 149-163. p. 157. Ver también Hochadel, n. 19, p. 211-252.
} 
objetivos $^{73}$. En este caso, con la polémica en los medios, el anuncio tuvo la intención de aumentar el eco de una conclusión, que el Hombre de Orce era un asno, que, sin esta difusión, hubiera pasado desapercibido publicado en una revista especializada.

Este ejemplo muestra cómo la publicación científica no es suficiente para establecer un nuevo conocimiento sino que, para validarlo, es «necesaria» una intervención en los medios. Así, los investigadores quieren «reforzar» sus afirmaciones intentando que primero sean aceptadas popularmente, para después ser validadas dentro de la comunidad científica ${ }^{74}$.

De esta manera, la prensa adquiere «poder» en la legitimación de los conocimientos. El interés público en la paleoantropología y la voluntad divulgadora de los científicos, en busca de apoyos y reconocimiento, llevan a esta ciencia a la prensa, que se convierte en otro espacio donde validar conocimiento.

\subsection{Los medios como espacio de debate}

Durante la polémica, los científicos utilizaron la prensa para expresar opiniones sobre sus investigaciones, o sobre conclusiones de otros científicos, sin pasar los controles que suelen pasar los artículos publicados en revistas especializadas. Entonces, la ciencia en los medios sigue los pasos y la lógica de éstos, distintos a los pasos y la lógica científica ${ }^{75}$. De esta manera, se establece un debate mucho más rápido y dinámico que el de las publicaciones científicas $^{76}$.

Como argumentó el propio Agustí en El País: «el debate debería haberse mantenido en los cauces de las publicaciones científicas pero el tratamien-

73. Un ejemplo clásico es: Lewenstein, Bruce. From fax to facts: Communication in the Cold Fusion saga. Social Studies of Science. 1995; 25 (3): 403-436.

74. Bucchi, Massimiano, When scientists turn to the public: Alternative routes in science communication. Public Understanding of Science. 1996; 5: 375-394.

75. Nieto-Galan, Agustí. Los públicos de la ciencia. Expertos y profanos a través de la historia. Madrid: Marcial Pons; 2011, p. 244.

76. El debate en las publicaciones científicas también se produce en el caso de Orce, por ejemplo: Gibert et al., n. 56 es una clara respuesta a Agustí; Moyà-Solà, n. 34 y Gibert; Campillo; García-Olivares, n. 59 contesta a Moyà-Solà; Köhler, n. 58. Habitualmente, estas réplicas se publican en las mismas revistas científicas que el artículo original. 
to periodístico del tema le ha dado una dimensión distinta ${ }^{77}$. El caso de Orce fue diferente y los mismos científicos lo detectaron y reconocieron.

Cuando El País publicó en portada la filtración de Marie-Antoinette de Lumley que ponía en duda la validez de VM-0, este periódico divulgó entre el gran público, incluidos los expertos, opiniones científicas que aún no habían sido publicadas en un medio especializado:

«tras la paciente tarea de limpiado de la cara interna del cráneo $[\ldots]$ se ha descubierto en la misma una pequeña cresta que [...] ha hecho temer que pueda no tratarse de un antepasado del hombre y que, [...] tiene todos los indicios de ser un équido ${ }^{78}$.

El día siguiente, en el mismo periódico, Gibert respondió a de Lumley con afirmaciones aún más «impenetrablemente» científicas:

«[La cresta] en nuestro cráneo es sencilla, los caballos la tienen doble. No tiene las características de lámina sino que es más bien en forma de uve. Las diferencias de la cresta del interparietal son las siguientes: Es doble en el ejemplar del IPH [L'Institut de Paléontologie Humaine]; es sencilla en un ejemplar joven del Laboratorio de Anatomía Comparada (LAC), pero, en ambos casos, presenta una forma que se agranda hacia la base, nunca la forma laminar del hombre de Orce» ${ }^{79}$.

Esta afirmación se encuentra, prácticamente igual, en el artículo que Gibert publicó el mes siguiente en las actas del congreso homenaje a Luis Siret ${ }^{80}$. En estos ejemplos vemos como Gibert articula su discurso teniendo en cuenta tanto la prensa como las publicaciones dirigidas a los expertos ${ }^{81}$.

En mayo de 1986, la revista Muy Interesante publicó una entrevista a María Teresa Alberdi, paleontóloga del Instituto de Geología del CSIC en Madrid que en aquellos momentos trabajaba en la geología de la región de

\footnotetext{
77. Redacción, n. 33.

78. Relaño, n. 15, p. 22.

79. Arroyo, Francesc. Josep Gibert considera que la hipótesis más fidedigna sigue siendo la del homínido. El País. 13 May 1984.

80. «Es doble en el ejemplar del IPH y sencilla en los ejemplares 313 y 72 del LAC. En el fragmento de Orce es sencilla». Gibert, Josep. El yacimiento de Venta Micena (Orce, Granada). Su importancia, acción antropogénica y características paleoantropologicas del fragmento de cràneo de Homo sp. In: Homenaje a Luis Siret. Sevilla: Consejería de Cultura de la Junta de Andalucía; 1984, p. 42.

81. Un ejemplo de discursos para diferentes audiencias es analizado en Fahnestock, n. 42, p. 27.
} 
Orce, en la que hablando sobre el fragmento de Orce afirma que «a juzgar por lo que ha salido en la prensa [...] se trata de una pieza algo rodada que estaba sin limpiar por dentro» ${ }^{82}$.

Los científicos hablaron en los medios para el gran público pero también para sus colegas, que recibieron información científica a través de éstos. La prensa fue entonces un lugar donde se habló de ciencia entre expertos, donde se discutió ciencia.

Después de la polémica inicial, empieza un debate científico en los medios de comunicación en el que intervienen, además de Gibert y los de Lumley, otros científicos, como Alberdi, Toro o catedráticos de paleontología, políticos, periodistas o incluso habitantes del propio Orce. Desde 1984 hasta 1987, cuando Moyà-Solà y Agustí publicaron el mencionado primer artículo que defiende que VM-0 no sería un homínido, el escenario de debate científico fue exclusivamente público. Además, los medios de comunicación cubrieron los congresos científicos convirtiéndolos en espacios de acceso más generalizado ${ }^{83}$. Más adelante, el debate continuó en las publicaciones científicas pero también lo hizo en los medios, desvaneciéndose así las fronteras entre lo científico y lo popular ${ }^{84}$.

\section{Conclusiones}

Después del hallazgo, los tres científicos descubridores consiguieron una plaza de investigadores en el Institut de Paleontologia de Sabadell ${ }^{85}$. Ser los descubridores del famoso Hombre de Orce fue clave en su consolidación profesional. Más adelante, Gibert acaparó las críticas al Hombre de Orce, que fue la «obsesión» de su vida, pero Agustí y Moyà-Solà, libres del peso de la controversia, continuaron unas exitosas carreras ${ }^{86}$. Así, el

82. Para un ejemplo del trabajo de Alberdi en la cuenca de Guadix-Baza: Alberdi, Maria Teresa; Bonadonna, Francesco Paolo, eds. Geología y paleontología de la cuenca de Guadix-Baza. Colección Trabajos sobre neógeno-cuaternario. 1989; 11. Torreiglesias, Manuel. Los Fósiles son patrimonio de la humanidad. Muy Interesante. 1986; 60: 129-131 (130).

83. Por ejemplo, la cobertura del Congreso de Southampton: Paredes, n. 30 y Moreno, n. 31 o para el de Orce ver nota 35.

84. Hilgartner, Stephen. The dominant view of popularization: Conceptual problems, political uses. Social Studies of Science. 1990; 20 (3): 519-539 (525).

85. Gibert, n. 1, p. 41

86. Entrevista Agustí, n.1 y para la «obsesión» de Gibert: Planas, n. 51. 
Hombre de Orce supuso un «puente», que ayudó a mantener el Institut de Paleontologia de Sabadell al principio de la democracia, entre la muerte del paleontólogo Miquel Crusafont, dominante durante el franquismo, y los descubrimientos de diversos fósiles de antropomorfos de Moyà-Solà, ya entrado el siglo XXI ${ }^{87}$.

A pesar de que la industria lítica hallada en la zona de Orce parece no dejar dudas acerca de la presencia de homínidos hace alrededor de un millón de años, el fragmento polémico sigue sin encontrar un consenso total entre investigadores. A la vez, los permisos de excavación en Orce siguen, al menos hasta hace poco, suscitando controversia ${ }^{88}$. Como hemos visto en la segunda parte de este trabajo, todo esto muestra cómo alcanzar un consenso entre científicos con opiniones diferentes, es casi imposible sin la intervención de cuestiones sociales como la autoridad o la credibilidad.

En 1976, mientras Gibert y Agustí descubrían el yacimiento de Venta Micena, en una sierra cerca de Burgos, Trinidad de Torres, doctorando de Emiliano Aguirre, desenterraba los primeros huesos de lo que sería el yacimiento de Atapuerca. Desde que en 1991 tomaron el control del yacimiento el actual triunvirato de directores, esta excavación se ha caracterizado por establecer una fructífera relación con los medios que han servido para «hacer una montaña mágica» ${ }^{89}$.

La voluntad divulgadora que los descubridores del Hombre de Orce mostraron durante el primer año después del descubrimiento, con apariciones en periódicos y revistas de divulgación, hace entrever que las investigaciones de Orce, que también podrían ser muy fructíferas, buscaban esta gran repercusión en los medios de comunicación. El caso de Orce fue entonces otro ejemplo de lo que se ha llamado «ciencia mediática» ${ }^{90}$.

Ahora bien, la principal diferencia entre el caso de Orce y estos otros ejemplos es, según mi entender, la opción utilizada por Marie-Antoinette

87. Para el papel de Crusafont durante el franquismo ver Florensa, en este dossier, y para MoyàSolà, por ejemplo, Antón, Jacinto. Pau, el abuelo en la basura. El País. 31 Jul 2005.

88. Por ejemplo: Ansede, Manuel. El «Hombre de Orce» seguirá bajo tierra. Público. 11 Ago 2011. Recientemente se ha divulgado el hallazgo de un diente de un homínido en la zona de Orce. Europa Press (Granada). Descubren en Orce el diente fósil de un homínido, que se convierte en el resto humano más antiguo de Europa. La Vanguardia. 1 Mar 2013.

89. Hochadel, n. 19 y n. 72

90. Nieto-Galán, n. 75, p. 243-273. Peter Weingart también ha desarrollado el concepto de «medialization of science» en varios de sus trabajos. Por ejemplo: Weingart, Peter. Science and the media. Research Policy. 1998; 27: 869-879. Hochadel, n. 19, p. 149-211. 
de Lumley para mostrar su desconformidad con los descubridores. El hecho que de Lumley escogiera El País convirtió a los periódicos no sólo en espacios donde se presentó ciencia al gran público sino en el lugar donde tanto la opinión pública como los expertos se informaron sobre el caso ${ }^{91}$. La controversia apareció exclusivamente en los medios públicos durante al menos tres años, hasta la publicación de Agustí y Moyà-Solà en Estudios Geológicos. Los periódicos no fueron entonces «simples aparadores» de información «fugaz», olvidada en pocos días, sino que tuvieron cierto «poder» dentro del «juego» científico.

Habiéndose trasladado el debate a los periódicos, su público se amplió así como el número de sus participantes. Además de los profesionales involucrados, intervinieron en la discusión otros científicos, periodistas o políticos. El papel de estos últimos fue muy importante. Al ser también un público presente en los medios, los políticos tomaron decisiones alrededor de las excavaciones en Orce basadas en la presencia pública de la polémica.

En varios ejemplos durante la controversia se aprecia como los científicos presentan y articulan su discurso y sus opiniones, tanto en los medios de carácter científico como en los medios de amplia difusión. Vemos entonces la dificultad de establecer los públicos específicos, expertos o profanos, de una publicación concreta. Los géneros de publicaciones se mezclan y reciben feedback los unos de los otros, siendo así difícil de encontrar una frontera clara entre ellos ${ }^{92}$.

Además, como ha mostrado Lewenstein en el caso de la fusión fría, el hecho de que los expertos se informen en la prensa o que ésta sirva de aparador de conocimiento científico no publicado anteriormente, muestra cómo para entender el modo en que se genera conocimiento científico hay que entender cómo se comunica este conocimiento de todas las formas posibles entre diferentes medios ${ }^{93}$.

91. Esto acercaría el Hombre de Orce al caso de la fusión fría, en el que los medios también fueron la fuente de información para los científicos. La diferencia con este caso estaría entonces en que este período de información a través de los medios fue mucho menor en el episodio de la fusión fría. Lewenstein, n. 73, p. 425-428.

92. Hilgartner habla de un continuo de géneros en el que diferenciar entre divulgación y ciencia es cuestión de grado, n. 84, p. 524-529.

93. Lewenstein presenta un modelo de comunicación científica «en red» dónde los medios de comunicación interaccionan de múltiples formas, siendo imposible distinguir entre la ciencia y su comunicación. Lewenstein, n. 73, p. 425-428. James Secord también plantea estudiar la ciencia como una forma de comunicación en Secord, James A. Knowledge in transit. Isis. 2004. 95: 654-672. 
Así pues, la historia del Hombre de Orce muestra un proceso científico que funciona de forma diferente: científicos, periodistas y opinión pública intervienen en el desarrollo de las investigaciones y el debate científico se produce en los medios públicos, siendo entonces la presentación de la ciencia en estos medios parte de la formación, legitimación y validación del conocimiento científico ${ }^{94}$.

\section{Agradecimientos}

Varias presentaciones públicas han alimentado enormemente este trabajo. Quisiera destacar la charla del 29 de noviembre de 2012, organizada por la Societat Catalana d'Història de la Ciència i la Tècnica, que sirvió para constatar que la controversia de Orce sigue viva 30 años después. Quiero dar las gracias por todas las sugerencias y apoyo recibido tanto de profesores como de alumnos del Centre d'Història de la Ciència (CEHIC-UAB). También quiero agradecer especialmente a Oliver Hochadel por guiarme en la historia de la paleoantropología y por la oportunidad de participar en este dossier; a José Pardo-Tomás por haber leído críticamente un borrador de este trabajo; a Clara Florensa su ayuda y ánimos y a mis padres por su apoyo y su lectura de mis escritos.

94. Como afirman Cloitre y Shinn las «expository practices play an active role in the knowledge production process». Shinn, Terry; Cloître, Michel. Expository practice: Social, cognitive and epistemological linkage. In: Shinn, Terry; Whitley, Richard, eds. Expository science. Forms and functions of popularization. Dordrecht: Kluwer; 1985, p. 31-60 (32). 


\section{Hominids, doubts and big headlines: The Orce Man controversy in the Spanish press (1983-2007)}

1.- Introduction. 2.-Orce Man: «The Finding of the Century» (or so it seems). 3.- International Congress of Human Palaeontology in Orce: «Josep Gibert was right» (or so it seems). 4.-Eight centimetres of polemic: anatomical characteristics of VM-0. 5.- "A distinct dimension»: the mass media and Orce Man. 5.1.-Dissemination and validation in the press. 5.2.- The media as a space for debate. 6.-Conclusions.

ABSTRACT: In June 1983, several Spanish newspapers announced the discovery of a cranial fragment of the genus Homo in Orce, Granada, which seemed a scientific revolution at the time. One year later, the same fossil was attributed to a donkey, as the newspaper El País reported. A strident controversy began: the famous "Orce Man» became the "Orce Donkey», the discoverers were criticized for their precipitation and the scientific debate moved to the mass media. Through an exhaustive study of the case in the Spanish newspapers, this article first explores how this dispute unravelled and its relationship with the historical context of palaeontology and palaeoanthropology in Catalonia and Spain. Secondly, this paper seeks to understand why it was impossible to reach a consensus among the scientists dealing with «Orce Man». Finally, analysis of the role of the press in the dispute sheds light on how the media are involved in the generation and validation of scientific knowledge. 